\title{
EDITORIAL
}

\section{REPRODUCTIVE HEALTH AWARENESS AMONG ADOLESCENTS}

By the end of 1997, there were 1.5 billion young people (between the ages of 10 and 24) in the world, 85\% of them living in the developing countries(1). These young people face numerous reproductive heath challenges worldwide. Cardinal among these challenges include: unsafe sex, unwanted pregnancies, unsafe abortion, HIV/AIDS and other classical (sexually transmitted diseases) increased vulnerability to sexual abuse and lack of access to reproductive health services. These challenges are related to lack of and/or could be ameliorated by improving reproductive health awareness. Attitudes of adults such as denial of the fact that adolescents are sexually experienced at very tender age negatively impacts on improvement of reproductive awareness among adolescents.

The age of sexual debuts(coitarche) varies considerably among countries and regions. In some countries, especially Asia and Latin America, median age at coitarche has increased while in others there has been a decrease over time. Average age of coitarche is 13 to 18 years(2,3). Early coitarche is associated with increased sexual activity. In some studies $50 \%$ to $90 \%$ of persons aged 10 to 24 years are sexually experienced $(2,4,5)$. Adolescents are tending to delay age at marriage. In United States of America, the age at marriage is about 24 years and 26 years for females and males respectively(6). The increase in age at first marriage and the decreasing age of coitarche has implications on the number of sex partners. Even where age of coitarche is increasing, age at marriage is increasing faster. It has been shown that the longer the interval between the first intercourse and the first marriage the more the number of sex partners a sexually activewoman or man is likely to have.

Sexual activity among adolescents may occur as part of experimentation, which characterises adolescent development. Young peoples sexual relations are often unplanned, sporadic and sometimes, the result of peer pressure or force. As shown in the study from Malawi, $87 \%$ of the adolescents who did not have sex were assaulted or forced in to it while $3 \%$ did it in exchange for money or gifts(2). Sexual relations typically occur before adolescents have gained experience and skills in self-protection, acquired adequate information about STD's, and before they can get access to health services and supplies such as condoms.

Each year more than one out of twenty adolescents contracts a curable STD, not including viral infections. By 1997, it is estimated that of the 333 million new annual STD's cases, at least 111 million occur in young people between under 25 years of age(1). Globally, more than half of all new HIV infections are among the 15-24 years age group(7). In most parts of the world, the majority of new infections are in young people between the ages of 15 and 24, sometimes younger. HIV incidence rates are six times higher in girls than in boys of the same age (8). Young girls are especially vulnerable for physiological, social and economic reasons. In general, new HIV infections in the younger age groups continue to rise even though the overall proportion of people living with HIV/AIDS is on the decline. However, in some countries, such as Thailand, Uganda and Zambia, there has been a decline in HIV prevalence among young people as a result of increased condom use(8).

One consequence of sexual activity is pregnancy. Several studies show that teen pregnancies are overwhelmingly unintended. Despite this, worldwide, more than $10 \%$ of all births are to women 15 to 19 years of age(1). The high number of teenage deliveries could be due to several factors including: lack of contraceptive use or access, the sporadic nature of teen sexual activities, and their ambivalence about pregnancy. Between 1995 and 1997, 105 of the Malawi adolescents receiving postabortal care had used a contraceptive method while $45 \%$ of the pregnancies among adolescents treated for abortion were unwanted(2). Twenty four per cent of males and $20 \%$ females sexually experienced adolescents in Ghana reported consistent use of condoms (9). The outcome of unwanted pregnancies is abortion. Adolescent abortions are estimated between 1 and 4.4 million per year, most of which are unsafe because they are performed illegally and under harzadous circumstances by unskilled practitioners (1). In Africa, $20 \%$ to $80 \%$ of all maternal deaths are abortion-related (10). A study from a teaching hospital in Nigeria showed adolescent maternal mortality ratio of 4863/ 100,000 live births, which was about two times that of the general population, 2152/100,000 live births.

Some of the challenges facing adolescents are related to level of education. Higher education is associated with better knowledge of reproductive health matters. As shown in the study from Malawi in this issue of the journal, more educated adolescents were more likely to know safe days of the menstrual cycle, and where menstruation comes from and to start sexual liaisons later(2). Other studies show good quality education fosters analytical thinking and better health habits(8). Sub-Saharan countries have high school drop out rates coupled with low enrolment, which means that few young people get any education at all. In the least developed countries, only $13 \%$ of the girls and $22 \%$ of the boys enrol for secondary education(1). Thus programmes that focus on secondary education are likely to reach a small proportion of the adolescents. There is need to develop appropriately designed reproductive health education programmes which start to pass relevant message from very early on in school 
life. There is genuine concern as to the content and depth of the message to be passed, with some religious groups being vehemently opposed to such a programme(family life education). From a human rights perspective, it is prudent to pass the appropriate reproductive health message. As to what the individual will do with the information, then, that becomes personal responsibility. Reassuringly, a review of more than 50 sex education programmes around the world found that young people are more likely to delay starting their sexual activity when they are provided with correct information about sexual and reproductive health (8).

Barriers to access reproductive health services include location, staff attitude, cost of the service, adolescents perception and attitudes. It has been shown that by removing cost barriers, increasing the number and type of providers, ensuring confidentiality, this could increase the number of adolescents accessing the service and thereby providing an opportunity to reduce unintended pregnancies and sexually transmitted infections (12). However, as shown from a study among Indian adolescents, other contextual considerations are important. In the Indian study, the limited mobility, lack of family and partner support, need for privacy to prevent stigma, led many of the adolescents to seek traditional providers services, even though safe options existed (13).

In conclusion, from the foregoing, the real problems teenagers pose, in terms of social and public health concerns are their failure to protect themselves against unwanted pregnancies and sexually transmitted diseases. There is thus a global need for adolescents and youth friendly reproductive health services, counselling of the youth on the sexuality, pregnancy, post-abortion issues and family planning, age appropriate sex education imparted with sensitivity to the needs of youth. Specifically, there should be programmes that enable young people to acquire life and negotiation skills, which are essential for making safe and informed choices. Adolescents need to have skills to counter threats of sexual coercion and force, refuse unwanted sex, insist on condom use and assert themselves in decisions on initiation of sexual activity and contraceptive use. The quality of reproductive health services need to address the concerns of the adolescents such as lack of privacy and confidentiality, mistrust and perceived judgmental attitudes among providers. The key contextual factors that influence adolescent behaviour need to be addressed. It is with great anticipation that we shall be expecting to read from this journal articles addressing some of the issues raised above. Certainly, the gesture of the Malawi government to allow adolescents to go back to school after pregnancy, if they so wish, is a commendable effort towards improving adolescent reproductive health, by ensuring the girls receive education. Such policies from other governments are anticipated, and will go a long way in improving reproductive health awareness and thereby the overall health of young people.

P. B. Gichangi, BSc (Hons), MBChB, MMed(Obs/ Gynae), Lecturer, Departments of Human Anatomy and Obstetrics and Gynaecology, College of Health Sciences, University of Nairobi, P.O. Box 2631, KNH 00202, Nairobi, Kenya

\section{REFERENCES}

1. WHO. Young people and sexually transmitted diseases. Http:/www.who.int/inf-fs/fact 186.html, 1997.

2. Lema, V.M. Reproductive awareness, behaviour and profiles of adolescents post abortion patients in Blantyre, Malawi. East. Afr. Med. J. 2003; 80:339-344.

3 Mwakagile, D., Mmari, E., Makwaya, C., et al. Sexual behaviour among youths at high risk for HIV-1 infection in Dar es Salaam, Tanzania. Sex Transm. infect. 2001; 77:255-259

4. Buga, G.A., Amoko, D.H., and Ncayiyana, D.J. Sexual behaviour, contraceptive practice and reproductive health among school adolescents in rural Transkei. S. Afr. Med. J. 1996; 86:523-527.

5. Glover, E.K., Bennerman, A., Pence, B.W., et al. Sexual health experiences of adolescents in three Ghanaian towns. Int. Fam. Plan. Perspect. 2003; 29:32-40.

6. Rodgers, W.L., and Thornton, A. Changing patterns of first marriage in the United States. Demography 1985; 22:265279.

7. UNAIDS. AIDS epidemic update, 2002.

8. UNICEF. Young people and HIV/AIDS opportunity in crisis. A joint report by UNICEF, UNAIDS and WHO, 2002.

9. Karim, A.M., Magnani, R.J., Morgan, G.T., and Bond, K.C. Reproductive health risk and protective factors among unmarried youth in Ghana. Int. Fam. Plan. Perspect. 2003; 29:14-21.

10. Otsea, K. The place of abortion care in safe motherhood programs. J. Obstet. Gynaecol. East Cent. Africa. 1993; 11:3-7

11. Airede, L.R., and Ekele, B.A. Adolescent maternal mortality in Sokoto, Nigeria. J. Obstet. Gynaecol. 2003; 23:163-165.

12. Brindis, C.D., Llewelyn, L., Marie K., et al. Meeting the reproductive health care needs of adolescents: California's Family Planning Access, Care, and Treatment Program. J. Adolesc. Health. 2003; 32:79-90.

13. Gantra, B., Hirve, S. Induced abortion among adolescent women in rural Maharashtra, India. Reprod. Health Matters. 2002; 10: 76-85. 